\title{
Relación entre ambiente, interacción, salud mental materna y el desarrollo psicomotor del niño mediante análisis de ecuaciones estructurales
}

Rolando Rivera González, ${ }^{1,2}$ Ignacio Méndez Ramírez, ${ }^{2}$ Miriam Figueroa Olea, ${ }^{1}$ Héctor Ávila Rosas, ${ }^{1,2}$ Antonio Sierra Cedillo ${ }^{1}$ y Karla Soler Limón ${ }^{1}$

\section{Introducción}

Las determinantes externas del desarrollo como la experiencia, las interacciones tempranas, los estilos de crianza, el involucramiento materno y otros factores psicosociales se han descrito de manera diversa en su forma de operar sobre el desarrollo. La influencia cambia según elementos complejos del entorno, como contextos económicos, sociales y culturales, la información y apoyos formales e informales disponibles. Entre los factores positivos relacionados al desarrollo y crianza se han descrito las interacciones y comunicación temprana, los ambientes estimulantes, la educación y salud mental materna. Las relaciones, por lo general, se han observado de forma aislada, o en diseños multivariados unidireccionales sin evaluar interacciones entre variables, multicolinealidad, ni senderos en las relaciones de determinación. Un ambiente social, emocional y físico desfavorable 
puede repercutir sobre los procesos de cuidado y las formas de relación del niño con el ambiente, constituyéndose en factores de riesgo psicosociales inmediatos que afectan el desarrollo alcanzado por el niño (Engle et al., 2007). El contexto y el sistema familiar se encuentran asociados con la adaptación individual, la cual puede incrementar o disminuir el impacto de los riesgos en el desarrollo (Lemelin, Tarabulsy y Provost, 2006). Entre los factores negativos para el desarrollo infantil, se enfatiza en la pobreza, escolaridad de los padres, hacinamiento, salud mental de los padres y familiares, condiciones de estrés social crónico, marginación social, violencia intrafamiliar y ausencia de redes sociales para el apoyo.

La salud mental de la madre es de los riesgos psicosociales más documentados junto con la depresión materna y otros problemas mentales, la exposición a la violencia o abuso y consumo de drogas durante el embarazo (Walker et al., 2011, Reichman, 2003). El efecto de los síntomas depresivos en la madre sobre las puntuaciones del desarrollo cognitivo han alcanzado efectos de 0.5 y 1.0 DS de diferencia sobre la medición del desarrollo (Engle et al., 2007).

Lamy et al. (2011) establecieron que las condiciones ligadas a la pobreza y la posición socioeconómica influyen sobre el desarrollo infantil. En niños saludables de 2 a 3 años en un contexto de pobreza, la sospecha de retraso del desarrollo según la escala de Gesell fue de $44.9 \%$, siendo las áreas de comportamiento adaptativo (56.8\%) y de lenguaje (63.1\%) las más comprometidas. Estudios sobre la influencia del estatus socioeconómico sobre los sistemas neurocognitivos encontraron que afecta más al procesamiento del lenguaje que a otros sistemas (Hackman, Farah y Meaney, 2010). Entre los elementos con mayor contribución para retraso en el desarrollo fue la presencia de hacinamiento (OR 3.7, IC 95\% 1.8-7.7) y la baja intensidad de estímulos en el ambiente familiar (Lamy et al., 2011). Las familias ubicadas por debajo de la línea de pobreza tienden a involucrarse menos en actividades que promuevan cognitivamente a sus hijos (Walker et al., 2007; Engle et al., 2007; Walker et al., 2011). 
La calidad de la crianza es hipotetizada para actuar como un mediador clave del vínculo entre la exposición del niño a la pobreza y el subsecuente desarrollo fisiológico, neurobiológico y cognitivo. La calidad del ambiente conduce a tipos de conductas de crianza que inician una cascada fisiológica que conduce a patrones de desarrollo que son apropiados o benéficos para ese medio ambiente (Meaney, 2010; Blair y Raver, 2012).

Por su parte, los procesos de interacción, vínculo y estabilidad emocional juegan un rol determinante en la mayoría de los casos. La falta de responsividad maternal constituye un riesgo para el desarrollo infantil. Se ha asociado con un apego inseguro, menores habilidades cognitivas y con el incremento de problemas conductuales (Engle et al., 2007). Se han descrito los efectos del apego (15 meses) sobre el desarrollo socioemocional, lingüístico y cognitivo directamente y en relación con los riesgos contextuales acumulados (36 meses) (Belsky y Pasco, 2002). En los países en desarrollo, sólo 10 a 41\% de los padres proveen materiales para estimular cognitivamente a sus hijos y de 11 a 33\% de los padres involucran activamente a sus hijos en actividades de estimulación cognitiva. Las familias ubicadas por debajo de la línea de pobreza tienden a involucrarse menos en actividades que promuevan cognitivamente a sus hijos (Walker et al., 2007; Engle et al., 2007; Walker et al., 2011).

Aunque se acepta que la desventaja psicosocial repercute sobre la crianza y el desarrollo, no todas las condiciones psicosociales muestran la misma capacidad de afectarlo, ni lo hacen con la misma intensidad (Walker et al., 2007). Por ejemplo, utilizando modelos de ecuaciones estructurales, se indicó que el efecto de la condición socioeconómica sobre el lenguaje y la memoria era estadísticamente diferente del efecto sobre otros sistemas. Se encontraron diferencias significativas en el sistema de lenguaje y el componente de memoria de trabajo y una tendencia significativa hacia la predicción del SES en el componente de control cognitivo (Farah et al., 2006). 


\section{Objetivo}

Determinar la relación entre la estimulación en el hogar, las interacciones madre-hijo, la salud mental materna y la condición socioeconómica con el desarrollo psicomotor del niño menor de 3 años de edad.

\section{Método}

Estudio prospectivo, transversal descriptivo de 843 niños y sus familias, pertenecientes al estrato socioeconómico bajo de tres comunidades de la República Mexicana, en un muestreo proporcional probabilístico para la prevalencia de alteraciones en el desarrollo y ajuste de covarianzas para las variables propuestas al modelo. Mediante análisis de ecuaciones estructurales se buscó las relaciones entre el ambiente y estimulación en el hogar (Inventario HOME), las interacciones cuidador-niño (NCAFS), el perfil de salud mental e IQ maternos (cGs de Golbert y Beta II-R) y la condición socioeconómica, respecto al desarrollo psicomotor. Todas las variables se presentaron en valores normalizados a media de 0 y DS de 1; se utilizó el software EQS 6.1.

\section{Resultados}

Se utilizaron 27 variables de las cuales cuatro fueron agrupadas en un primer factor que representaba el desarrollo psicomotor del niño, seis correspondían a cada área del Inventario HOME, conformando un segundo factor denominado HOME. Otras 6 variables, que representaban las subescalas del NCAST, conformaron un tercer factor. Un cuarto factor agrupó variables relacionadas con la salud mental y calidad de vida maternos evaluados con el Cuestionario General de Salud de Golbert (cGs-Golbert) y el coeficiente intelectual de la madre evaluado con la prueba BETA-II R, quedando como variables aisladas en el modelo: la 
edad y género del niño, la edad y escolaridad de la madre, y la condición socioeconómica de la familia.

Formados los cuatro factores iniciales, se le asignó al primero el carácter de variable dependiente general para todo el modelo y a los factores 2, 3 y 4, el de variables independientes, con efectos directos al factor 1 . El factor 4 , salud mental y calidad de vida materna, no se ajustó a los datos del modelo con efectos directos.

Se estableció la relación entre los factores 2, 3 y 4 entre sí, mejorando el ajuste de los datos al modelo, por medio de los índices de modificación de ajuste de Wald para eliminar variables y los Multiplicadores de Lagrange por Máxima Verosimilitud para agregar variables o relaciones entre ellas. Se estableció la continuidad del factor 4 en el modelo, pero con un efecto indirecto (mediado por F2) sobre el factor 1 y directo sobre los factores 2 y 3; además, se estableció que la Variable CI materno debía eliminarse del factor 4, quedando como variable individual.

El factor 1 se organizó según el agrupamiento de las 4 áreas del desarrollo descritas (Alpha de Cronbach $=.84$ ). El área que representó mejor el factor 1 (desarrollo psicomotriz) como variable dependiente $u$ endógena en el modelo fue la cognitiva-lenguaje con saturación de .91, y la que menos lo hizo fue la motriz gruesa con un coeficiente de .61. Entre los errores de las áreas COG-LE y la MAM-PR existió una correlación negativa de -.59 $(p<.05)$.

El factor 2-HOME estuvo representado por todas las áreas (alfa Cronbach $=.62$ ). Los coeficientes más altos para el factor fueron dados por las áreas involucramiento, material y variedad (con coeficientes de .77, .72 y .39, respectivamente).

El factor 3-NCAST, conformado por las seis subescalas del NCAST (alpha .85), las subescalas fomento al crecimiento emocional, responsividad al cuidador y fomento cognitivo fueron las que representaron mejor este factor con índices de $.89, .80$ y .74 , respectivamente.

El cuarto factor propuesto en el modelo lo conformaron los cuatro componentes del cGs-Golbert (Alpha .76); en él, las áreas ansiedad y síntomas somáticos fueron las que representaron mejor el factor. 
En el modelo general se pretende establecer la relación entre los factores independientes $u$ exógenos con el dependiente o endógeno F1 (desarrollo psicomotor). Dos de los tres factores propuestos al modelo mostraron efectos directos, el Factor2- HOME y el Factor 3-NCAST. De ambos, el Factor HOME fue el mejor asociado con un coeficiente de sendero tres veces mayor (.33) que el del Factor NCAST (.11). El Factor 4 (cGs-Golbert) no mostró efectos directos importantes, pero sí se expresaron efectos indirectos discretos a través del Factor 2-HOME con un coeficiente de -.06).

Cuando se estableció el ajuste del modelo a los datos y permanencia de los factores en el modelo, se incorporaron cinco variables generales: la edad y género del niño, la edad y escolaridad de la madre y la condición socioeconómica de la familia. De las variables generales, sólo la variable cı materno pudo incorporase al modelo, las otras variables no mostraron efectos importantes o su presencia generaba efectos de colinealidad, reduciendo la validez y significancia del modelo. La incorporación de variables aisladas o agrupadas en factores se evaluó recurrentemente utilizando índices de modificación de ajuste de Wald para eliminar variables y los Multiplicadores de Lagrange por Máxima Verisimilitud para agregar variables o relaciones entre ellas. El cl materno mostró importante asociación con los factores 2 y 3 , mucho más fuerte con el factor 2-HOME con un efecto directo (.54) que con el factor 3-NCASt (.16), aunque el análisis sugirió, además, relaciones directas con las subescalas Crecimiento Emocional (-.14) y Crecimiento Cognitivo (.24) del NCAST (a los que se agregaron efectos indirectos de .13 y .01, respectivamente). El efecto total de la variable cı materno sobre el F1-desarrollo fue de .19, logrado de forma indirecta (mediada por los factores 2 y 3), pero siendo el segundo en relevancia para explicar el F1-desarrollo después del HOME.

\section{Conclusiones}

Tres factores presentaron un efecto sobre el desarrollo: Inventario HOME, NCAST y el IQ materno, mediante efectos directos e 
indirectos; la salud mental y condición socioeconómica sólo se asoció mediante efectos indirectos a través de la estimulación en el hogar y la interacción cuidador-niño. Los factores psicosociales operan sobre el desarrollo interactuando de manera compleja. El desarrollo cognoscitivo y motriz fino se asoció más con las variables psicosociales.

\section{Referencias}

Blair, C. y Raver, C.C. (2012). Child Development in the Context of Diversity Experential Canalization of Brain and Behavior. American Psychologist, 67(4), 309-318.

Belsky, J. y Pasco, R.M. (2002). Infant-Mother Attachment Security, Contextual Risk, and Early Development: A Moderational Analysis. Development and Psychopathology, 14, 293-310. Engle, P.L., Black, M., Behrman, R., Cabral de Mello, M., Gertler, P., Kapiriri, L. Martorell, R., y Young, M. (2007). Strategies to Avoid the Loss of Developmental Potential in More than 200 Million Children in the Developing World. Lancet, 369, 229-242.

Farah, M.J., Shera, D.M., Savage, J.H. y Betancourt, L., Giannetta, J.M., Brodsky, N.L. y Hurt, H. (2006). Childhood Poverty: Specific Associations with Neurocognitive Development. Brain Research, 1110(1), 166-174.

Hackman, D.A., Farah, M.J., y Meaney, M.J. (2010). Socioeconomic Status and the Brain: Mechanistic Insights from Human and Animal Research. Nature Reviews Neuroscience, 11(9), 651-659.

Lamy, F., Medeiros, S.M., Carvalho, Z., Lopes Moreira, M.E. (2011). Ambiente domiciliar e alterações do desenvolvimento em crianças de comunidade da periferia de São Luis - MA. Ciência \& Saúde Coletiva, 16(10), 4181-4187.

Lemelin, J.P., Tarabulsy, G.M. y Provost, M.A. (2006). Predicting Preschool Cognitive Development form Infant Temperament, Maternal Sensitivity and Psychosocial Risk. Merril Palmer Quarterly, 52(4), 779-806. 
Meaney, M. (2010). Epigenetics and the Biological Definition of Gene X Environment Interactions. 81, 41-79.

Reichman, N. (2003). Effects of Psychosocial Risk Factors and Prenatal Interventions on Birth Weight: Evidencefrom New Jersey's Health Start Program. Perspectives on Sexual and Reproductive Health, 35(3), 130-137.

Walker, S., Wachs, T., Carter, J. y The International Child Development Steering Group. (2007). Child Development: Risk Factors for Adverse Outcomes in Developing Countries. Lancet, 13(369), 145-15.

Walker, S., Wachs, T., Grantham-McGregor, S.M., Maureen, B., Nelson, C., Huff Man, S. y Richter, L. (2011). Inequality in Early Childhood: Risk and Protective Factors for Early Child Development. Lancet, 378(9799), 1325-1338. 